\title{
Pour une meilleure prise en compte de la faune ichtyaire à l'occasion des aménagements de rivières
}

\author{
J.J. Demars \\ Conseil Supérieur de la Pêche \\ Délégation Régionale pour l'Auvergne et le Limousin
}

\section{La faune ichtyaire, ses relations avec le milieu}

Pour le poisson le milieu aquatique ne se limite pas seulement à l'eau, il englobe également le contenant, permanent ou occasionnel : fond du lit, berges au-dessus et sous l'eau, zones d'inondations. Tous ces éléments demeurent sous l'influence des caractéristiques du bassin versant : nature du sous-sol, couverture végétale, latitude, longitude, altitude, qui régissent la qualité originelle de l'eau, sa quantité, son mode de passage vers la rivière, son écoulement dans le lit, la pente de ce dernier ainsi que sa couverture sédimentaire, la forme des berges et le développement de la ripisylve. En outre il est évident que le milieu aquatique est sujet à des fluctuations et modifications naturelles plus ou moins rapides, et donc plus ou moins perceptibles par l'homme.

L'examen du cycle de vie d'un poisson très répandu, la Truite commune, illustre les liens reliant l'animal à son habitat. Il s'agit d'une espèce qui acquiert un comportement territorial dans les 10 jours qui suivent son émergence des graviers au sein desquels elle est née (Heland, 1971). Une hiérarchie s'installe qui permet la fixation des sujets dominants sur des emplacements particuliers situés derrière un obstacle. Un comportement de défense du territoire élimine les individus dominés. Ce sont des éléments physiques qui marquent les limites des territoires et les individus choisissent leur site de préférence là où il y a le plus d'obstacles et d'abris. Ce comportement demeure durant toute la vie de l'animal qui en grandissant acquiert un domaine de plus en plus grand et qui en outre recherche lors de la reproduction certaines zones bien caractérisées: graviers $(\varnothing 10$ à $50 \mathrm{~mm})$, profondeur $(<50 \mathrm{~cm})$, vitesses $(20$ à $70 \mathrm{~cm} / \mathrm{s})$, percolées par l'eau et relativement stables (HERMANSEN, 1985). Cela conduit la Truite à réaliser des migrations liées à la reproduction. Plusieurs auteurs, dont Huet et Timmermans (1979), ont montré que l'on pouvait observer des déplacements de géniteurs vers l'amont avant la ponte, suivis après la reproduction de mouvements dans le sens inverse, d'abord par ces mêmes géniteurs, ensuite par des juvéniles. Les crues influencent nettement l'importance de ces phénomènes qui peuvent exister aussi bien au sein d'un cours d'eau qu'entre celui-ci et un petit tributaire.

La nourriture est essentiellement composée de larves d'insectes aquatiques inféodées aux associations vitesses de l'eau — taille des sédiments, et qui colonisent souvent la face inférieure cachée du substrat. La quantité et la qualité de ces invertébrés sont dépendantes de la diversité de leur habitat. Ainsi les sédiments fins ou bien ceux qui présentent une surface lisse parcourue par un courant d'eau très rapide constituent des milieux pauvres.

Nombreuses sont les études scientifiques qui démontrent les relations entre l'importance des populations de poissons et la diversité et l'adéquation de la structure physique du

\section{Taking into account fish populations when developing rivers}

Fish populations are distributed according to the characteristics of the environment and the requirements of the various species. Some indications show a certain variability of populations related to the fluctuations of parameters in the ecosystem. The examination of vital cycles shows up the specific needs and the consequences of natural or anthropical modifications of the environment. Some examples show the consequences of some developments on fish populations. Consideration of fish requirements has apparently changed favourably over the last few years. More numerous on site tracking has, however, led to less empiricism which usually prevailed on certain occasions in waterway upkeep. No section of the aquatic environment can be considered in isolation out of the context of the catchment area. 
lit. Ceci est particulièrement accusé pour les salmonidés. Parmi celles-ci on retiendra celle de LewIs (1969), qui a montré qu'il existe une relation étroite entre la densité de truites d'un ruisseau et plusieurs paramètres: surface, volume, profondeur, vitesse du courant et importance des abris ; ce dernier élément apparaissant comme le plus explicatif de la relation.

L'évaluation de la valeur ichtyaire d'un hydrosystème peut être approchée par l'analyse de l'habitat ou plus directement par l'examen des populations de poissons : stock en place, production annuelle, prélèvements halieutiques.

En matière d'estimation de la qualité physique du milieu les méthodes d'appréciation sont extrêmement peu nombreuses. MILNER (1985) indique qu'en GrandeBretagne l'évaluation quantitative de l'habitat n'a pas constitué une partie importante dans la gestion des milieux aquatiques et de la pêche. Il pense cependant que devant l'augmentation des pressions sur l'utilisation des cours d'eau existe un besoin de développement de ces méthodes. En France, la méthode des micro-habitats commence à être employée pour prévoir l'impact des réductions des débits ; néanmoins elle demeure encore en cours d'amélioration. Ce manque de méthodes contraste avec les évaluations quantitatives que peuvent fournir les aménageurs quant à leurs besoins, par exemple en matière de débit ou de section pour l'évacuation des crues.

Plusieurs techniques d'échantillonnage permettent de connaître la diversité ichtyaire présente dans une station : pièges, filets, pêche électrique, prélèvements par les pêcheurs. L'expérience montre que la combinaison des méthodes de prélèvement et leur répétition dans le temps limite les biais d'échantillonnage en grands cours d'eau. L'outil pêche électrique est particulièrement indiqué pour des recensements exhaustifs dans les petits cours d'eau. Ceux-ci abritent souvent des populations de Truite. Comme cette espèce est particulièrement appréciée par les pêcheurs, de nombreuses données ont été accumulées. Leur exécution n'a pas, au moins jusqu'à cette année, répondu à une programmation inscrite dans un cadre national de connaissance et de surveillance de la faune aquatique. L'analyse globale de ces données n'a pas non plus toujours été envisagée. Les références sur la répartition des populations, leur importance et leur évolution ne sont donc pas toujours disponibles. Cependant certaines connaissances ont été acquises. De nombreux auteurs ont montré que selon les besoins des espèces: thermiques, morphodynamiques et trophiques, existe une organisation spatiale qui fait que se retrouvent ensemble celles des espèces qui présentent les mêmes exigences. Ainsi Hutet (1949) a défini une classification longitudinale des eaux courantes en fonction d'une règle des pentes et des largeurs : zones à Truite - à Ombre - à Barbeau - à Brême (d'amont en aval). VERNEAUX (1981), par analyse statistique de 450 stations d'échantillonnage de poissons, rend compte de la composition des peuplements ichtyologiques potentiels associés à 10 types de milieux rencontrés entre les sources et les zones inférieures du réseau hydrographique. Il précise aussi l'amplitude et le preferendum typologiques de 31 espèces pisciaires et il indique qu'une espèce résiste d'autant plus à des changements de son milieu qu'elle se trouve proche de son preferendum. La diversité ichtyaire observée dans une station fournit une image de la qualité du milieu. Globalement d'amont en aval le nombre d'espèces augmente.

Quelques analyses d'inventaires exhaustifs de populations de truites communes permettent de constater, en zone de moyenne montagne typiquement salmonicole, divers types de fluctuations sur un même cours d'eau (C.S.P., rapports internes non publiés). Il apparaît souvent une diminution de la densité et même de la biomasse présentes par unité de surface à mesure qu'augmentent la distance à la source et la largeur. En une même station, d'une année sur l'autre, l'importance de la population peut tout aussi bien varier de manière prononcée que demeurer stable. Dans un même département où la nature géologique et la vocation agricole des bassins versants sont différentes, on observe, associées à ces entités, des peuplements eux aussi différents en densités et en biomasses. Ces remarques permettent d'entrevoir que les populations de ce salmonidé sont bien sous la dépendance non seulement des caractéristiques du milieu mais également des fluctuations naturelles auxquelles elles sont soumises. Il peut aussi être pensé qu'une seule photographie, isolée dans le temps, reste insuffisante parfois pour apprécier une population de poissons.

Les perturbations anthropiques du milieu physique, en transformant l'habitat du poisson risquent, d'en modifier l'importance et la structure des peuplements. Ces actions peuvent concerner l'eau: quantité, modalités d'écoulement; le lit: nature et diversité des sédiments ou de la flore, profil en long, section en travers; les berges : pente, caractéristiques de la surface en contact avec l'eau, développement et nature de la ripisylve. La modification d'un ou de plusieurs paramètres du milieu directement dans le cours d'eau, à proximité ou dans une zone plus éloignée dans le bassin versant, peut avoir des conséquences sur les populations ichtyaires non seulement au droit de la station où elle s'exerce mais aussi plus en aval puisqu'un cours d'eau présente un double flux liquide-solide entrant directement dans la valeur de l'habitat. Du fait de l'existence de poissons migrateurs une altération du milieu d'une partie située en aval dans le réseau hydrographique peut engendrer des modifications sur les peuplements de poissons présents plus en amont.

\section{Quelques exemples, variés de transformations de l'habitat et de leurs conséquences sur la faune ichtyaire}

\subsection{Modifications du débit et de l'écoulement}

La dérivation d'une part importante du débit, parfois associée à un stockage, provoque plusieurs types de problèmes et qui se rapportent : à l'obstacle physique que représente le barrage, aux perturbations de l'habitat dans les zones soumises au débit réservé et parfois à des éclusées, 
au remplacement d'une zone courante de l'hydrosystème par une retenue.

Des connaissances techniques fiables ont été acquises en matière de circulation des poissons vers l'amont au moyen de dispositifs spécifiques: les échelles à poissons. Des progrès restent cependant à faire dans 2 domaines: le détournement des migrateurs dès les canaux de fuite des usines hydroélectriques où ils sont attirés et la dévalaison des juvéniles.

L'examen des stocks en place de truites dans une dizaine de sites affectés par des usines hydroélectriques fonctionnant au fil de l'eau a montré des modifications apparentes fort diverses (DEMARS, 1985). Il existe une relation entre l'évolution du peuplement dans les sections à débit réduit par rapport à celui dans des sections témoins et l'importance du débit réservé par rapport au module. Cette corrélation est encore plus nette quand est pris en compte le prélèvement opéré au projet de l'usine. Dans la majorité des cas est observé un stock de truites (densité, biomasse) moindre dans la section dérivée. Ce phénomène est d'autant plus accusé que le prélèvement de débit est élevé et que le débit réservé est faible (de $3 \%$ à $18 \%$ du module). Dans certains autres cas cependant cette situation n'apparaît pas (débit réservé de $11 \%$ à $15 \%$ du module). La fixation d'un débit réservé, exprimé sous la forme d'un pourcentage du module et qui serait partout identique ne tiendrait pas compte des spécificités morphodynamiques de chaque site. Une telle valeur peut être une base de réflexion. Cependant un retour d'expérience, c'est-à-dire des suivis des populations ichtyaires sur le terrain, doit être de nature à apporter une aide pour les décisions futures en matière de débit réservé.

CRISP (1985), en Angleterre, a montré que la régulation d'un cours d'eau salmonicole par la mise en place d'une retenue, a eu une incidence favorable sur les peuplements de Truite. Les débits inférieurs à $10 \%$, du module ainsi que ceux supérieurs à 5 fois le module sont supprimés. Les températures de l'eau présentent des fluctuations annuelles et journalières amoindries. Dans la rivière en aval, à moins de $1 \mathrm{~km}$ du barrage est notée, au cours des années postérieures à la mise en eau, une sensible augmentation de la densité de truites $(40 \%)$. Ce même phénomène a aussi été retrouvé dans plusieurs affluents du réservoir (pas tous cependant); là c'est plus spécifiquement la densité des juvéniles qui a augmenté. Dans la retenue, il n'y a pas eu de modification des espèces.

Beaucoup d'hydrosystèmes français sont le siège de nombreux plans d'eau à vocation hydroélectrique (grandes et moyennes rivières) ou de loisirs : étangs touristiques publics ou étangs privés (petites rivières et ruisseaux) construits au coup par coup sans gestion d'ensemble du bassin. Lorsque, comme c'est souvent le cas, les populations ichtyaires originales sont salmonicoles, il est presque toujours constaté leur disparition et leur remplacement par des cyprinidés et carnassiers inféodés aux eaux calmes.

\subsection{Modifications du lit et des berges des cours d'eau}

Réalisées trop souvent dans un seul souci, celui de l'éva- cuation de l'eau ou de l'extraction des sédiments, ces opérations ont occulté la dimension biologique de la rivière au profit d'un impératif hydraulique local et à court terme. Cette absence de prise en compte des intérêts piscicoles s'avère désastreuse pour la faune aquatique comme en témoignent quelques exemples de suivis.

Le Litrou, petit cours d'eau de la plaine de Limagne (63), a été curé, recalibré, et reprofilé au cours de l'hiver 1979 pour améliorer l'évacuation des eaux de ruissellement et de drainage. Le nouveau lit, sensiblement plus rectiligne et raccourci de plus de $20 \%$, offre un profil en travers trapézoïdal plus large et un profil en long uniforme ponctué de seuils en béton infranchissables pour les poissons. Une étude technique du CTGREF indique qu'à débit égal les profondeurs passent en étiage de $16,3 \mathrm{~cm}$ en moyenne (extrêmes : 7-43) à $8,4 \mathrm{~cm}$ en moyenne (extrêmes : $5-20)$ tandis que les vitesses augmentent de $17,2 \mathrm{~cm} / \mathrm{s}$ en moyenne (extrêmes : 6-46) à 23,1 cm/s (extrêmes : 11-39). La densité des invertébrés aquatiques, source importante de nourriture pour les poissons, est trois fois moindre dans la partie chenalisée du ruisseau que dans la partie amont non modifiée. Les populations de poissons ont été évaluées en plusieurs occasions (CUINAT et collaborateurs, 1982) avant la réalisation des travaux puis dans les années qui ont suivi leur exécution. Le nombre d'espèces chute de 14 à 4 immédiatement après l'aménagement ; 8 ans plus tard il demeure encore inférieur : 12 espèces, cependant tous les carnassiers Brochet - Perche - Lotte ont disparu alors que de nouveaux arrivants, peu appréciés des pêcheurs, ont fait leur apparition : Epinoche - Carassin - Perche soleil. Les biomasses ont très sensiblement régressé passant de $150 \mathrm{~kg} / \mathrm{ha}$ avant les travaux à $20 \mathrm{~kg} / \mathrm{ha} 3$ ans après, et de plus ces poissons sont des animaux de petite taille ou inintéressants pour les pêcheurs (Loche - Goujon). Huit ans après, la biomasse est remontée à $97 \mathrm{~kg} / \mathrm{ha}$, cependant elle reste dominée par des individus plutôt de faible dimension (Goujon). La population ichtyaire n'est donc toujours pas redevenue similaire à celle originelle et, même si une certaine hétérogénéité réapparaît dans le milieu physique, le cours d'eau conserve son lit raccourci et demeure enfermé à l'intérieur de berges trop hautes.

Huet et TIMMERMANS (1976) constatent des conséquences tout à fait semblables dans 3 petits cours d'eau (largeur originelle inférieure à $10 \mathrm{~m}$ ) en Belgique; de 3 à 12 ans après l'aménagement :

— régression de 20 à $60 \%$ du nombre d'espèces ;

— diminution de la biomasse de 10 à $50 \%$ affectant encore plus intensément la fraction pêchable (les grands poissons);

— perte de linéaire de rivière de 25 à $50 \%$ selon les cas ; - élargissement du lit, profondeur d'eau faible, écoulement uniforme, absence d'abris et mauvaise colonisation du milieu par la végétation aquatique sauf les algues, constituent les principales modifications du milieu; - régression de la population ichtyaire dans les sections de rivières non concernées par les travaux mais recevant le flux de m.e.s. car situées en aval.

De nombreux auteurs américains ont montré des évolutions similaires. TARPLEE (1971) a travaillé sur 28 stations 
indemnes et sur 46 autres transformées de 22 cours d'eau de Caroline du nord. Il fait notamment état de températures estivales plus élevées dans les rivières recalibrées et attribue ce phénomène à la suppression de la ripisylve. Il note également qu'il faut environ 15 ans sans autre agression pour qu'un cours d'eau retrouve sa diversité spécifique piscicole. Dans une autre région, en Alabama - Mississipi, ARNER (1976), constate que cinquante ans après les travaux de chenalisation l'hydrosystème n'a pas encore retrouvé la totalité de ses potentialités piscicoles.

On assiste maintenant en moyenne montagne, au moins, à la réalisation de très nombreux reprofilages, curages et recalibrages sur les têtes du réseau hydrographique, lesquelles sont caractérisées par des dimensions très restreintes (souvent de largeur inférieure à 1 mètre) et par une population nettement salmonicole avec parfois une vocation accusée de zone de reproduction. Il faut donc craindre de nouvelles nuisances pour certaines espèces comme la Truite ou l'Ecrevisse indigène. A notre connaissance, aucune étude n'a été conduite pour connaître les effets de cette modification de l'habitat (abris, profondeurs, vitesses, ripisylve) sur la faune aquatique associée ou de proximité, ainsi que sur la ressource en eau.

L'exploitation des sédiments directement dans le lit mineur des cours d'eau français a diminué mais ne paraît pas avoir totalement disparu. Clavel (1977) a décrit les effets de ces extractions sur la physico-chimie de l'eau, la granulométrie et la topographie du fond des cours d'eau, les macro-invertébrés aquatiques et les poissons. La transformation de l'habitat, soumis à un important flux de m.e.s., induit des nuisances par colmatage des œufs des poissons, ainsi qu'une modification de l'importance relative des espèces accompagnée d'une régression des densités et biomasses. Les perturbations du milieu physique, uniformisation de l'habitat et apparition d'obstacles à la circulation des poissons, ne cessent pas de facto avec l'arrêt des extractions. A l'évidence ces travaux ont été autorisés et souvent exécutés sans intégration du problème de la préservation de l'environnement. Ce dernier a été pris en compte parfois localement et postérieurement après constatation des nuisances : mise en place d'échelles à poissons, restauration de communications entre certaines annexes hydrauliques et la rivière principale.

Puisque l'on attribue à la dégradation de l'habitat des appauvrissements de diversité ichtyaire et/ou des diminutions des densités et des biomasses, on est en droit de penser que des aménagements qui provoquent une restauration de l'hydrosystème, considéré ici comme milieu de vie de la faune aquatique, sont à même d'améliorer les populations piscicoles.

BAUDET et MOUILLE (1981) montrent qu'un aménagement hydraulique de la Mortagne (88-54) destiné à réduire les inondations a permis d'améliorer la situation ichtyaire en intégrant l'aspect environnement. Les travaux ont visé à :
- nettoyer le lit des embâcles, végétaux notamment, qui l'obstruaient ;

- conserver l'hétérogénéité de l'habitat en maintenant un tracé sinueux, un profil en long comportant seuils et mouilles, un profil en travers diversifié ;

- limiter au maximum les coupures de méandres;

- n'élargir que sur une rive là ou cela était indispensable ;

- recréer un lit mineur sinueux dans un tronçon déjà recalibré sévèrement.

Il en a résulté une amélioration de la diversité faunistique, associée à une augmentation de stocks, qui la rapproche de celle attendue avec le niveau typologique du site.

Cette démarche est aussi celle proposée par NunNALly et Keller aux Etats-Unis (1979). En conservant une certaine ripisylve, en limitant les coupures de méandres et les berges néoformées, en respectant des sections en travers dissymétriques dans les courbes, en protégeant par enrochement et végétalisation les rives concaves, et en conservant une bonne partie de la diversité du profil en long, les travaux aboutissent à une dépense moindre et conduisent à une meilleure stabilité du lit tout en limitant les impacts sur la faune aquatique.

Il faut cependant constater le peu de données biologiques disponibles relatives à l'impact de ces méthodes de restauration des cours d'eau.

HuNT $(1969,1971)$ a étudié les conséquences d'un aménagement ayant porté sur 1700 mètres de rivière à Omble de fontaine. Les matériaux employés : madriers, blocs, terre végétale et engazonnement sont disposés en déflecteurs dans le lit de manière à en réduire la largeur jugée trop importante, tout en créant des abris : anfractuosités dans les blocs et sous-berges artificielles (WHITE et BRYNILDSON, 1967). L'étude a porté sur l'analyse des paramètres de l'habitat et des populations de poissons durant trois années avant les travaux et trois années après les travaux, et des comparaisons ont été faites entre la zone aménagée et une zone non aménagée située immédiatement en aval. La transformation du milieu se manifeste par une nette diminution de la surface du cours d'eau et par une régression des sables et limons au profit des sédiments plus gros. Dans le même temps le nombre et la surface des mouilles sont très sensiblement multipliés, de même que l'importance des abris permanents en sous-berge. Le stock en place contrôlé augmente très sensiblement grâce à une meilleure survie hivernale et ce sont particulièrement les poissons les plus grands qui bénéficient le plus de cet aménagement. En résultat la production annuelle et la récolte prélevée par les pêcheurs sont grandement développées. La finalité de ces travaux est inverse de celle des recalibrages-reprofilages :

- augmentation de la profondeur d'eau;

- augmentation des abris ;

— réduction de la surface mouillée ;

— diversification du profil en long du fond.

Dans une rivière à Truite du Massif-Central (Pontajou, 43) à pente modeste, l'eau serpente en érodant les berges 
faites de tourbe et de terre. Le fond offre peu de sédiments grossiers de diamètre supérieur à $25 \mathrm{~cm}$ et les abris pour les poissons sont dus pour l'essentiel à quelques sousberges en dehors de la présence de végétation aquatique en fin de printemps et en été. La protection des berges a consisté en la mise en place d'enrochements et de blocs (diamètre allant de $25 \mathrm{~cm}$ à plus d'l mètre) dans le lit mineur sur l'équivalent d'une rive entière dans les parties concaves affouillées. La comparaison des stocks de Truite commune entre 2 stations aménagée et non aménagée ayant la même pente $(4 \%)$ indique :

— une densité globale forte et similaire dans les 2 stations ;

- une plus forte proportion d'alevins dans la station non aménagée ;

- davantage de sujets de grande taille dans la station aménagée; et notamment $75 \%$ en plus de truites dépassant la taille légale de capture dans la zone ayant reçu les enrochements ;

- une biomasse de poissons ayant la taille légale de capture dans la station aménagée de $92 \%$ supérieure à celle de la station sans enrochement. Ces données (document CSP, 1991) mériteront d'être précisées sur plusieurs années. Elles laissent entrevoir un effet bénéfique de l'augmentation des abris disponibles; les enrochements fournissant un nombre très important de caches par les anfractuosités qu'ils créent. A titre d'illustration dans la station non aménagée $(108 \mathrm{~m} \times 4,9 \mathrm{~m})$ sont dénombrés 39 blocs de longueur comprise entre $25 \mathrm{~cm}$ et $90 \mathrm{~cm}$; dans la station aménagée en totalité sur une rive $(107,5 \mathrm{~m} \times 4,3 \mathrm{~m})$ existent 242 blocs d'une longueur comprise entre $25 \mathrm{~cm}$ et $150 \mathrm{~cm}$. L'aménagement hydraulique a, ici, respecté l'habitat du poisson, plus encore il semble l'avoir amélioré.

\subsection{L'entretien de la ripisylve}

Depuis une vingtaine d'années ont été développés des travaux dits d'entretien ou de restauration des rivières se rapportant pour l'essentiel à la végétation terrestre, qu'elle soit vivante ou morte et située dans ou sur les rives des cours d'eau. De nombreux documents qu'ils soient écrits ou visuels ont été édités par divers auteurs ou organismes (Ministère de l'Environnement, Agence de Bassin, CEMAGREF, etc...). Beaucoup traitent de la problématique, de la conception et de l'exécution des travaux après exposé des principes retenus dans la réflexion. Très rares sont ceux qui témoignent des conséquences réelles de ces entretiens sur la faune ichtyaire.

Les constatations visuelles faites avant les travaux peuvent être fort variées d'une rivière à l'autre en fonction des caractéristiques naturelles du lit et de l'état d'abandon. En théorie on peut retenir comme conséquences importantes sur les populations piscicoles :

- des abris nombreux occasionnés par les embâcles;

— une protection de la forêt galerie vis-à-vis de l'échauffement thermique estival ;

- un apport important de matière organique végétale terrestre ;
- une limitation de la quantité de lumière (et donc d'énergie) arrivant dans le milieu aquatique avec, en corollaire, la réduction voire l'absence de la végétation aquatique; - un écoulement perturbé sans beaucoup de diversité, accompagné d'une importante sédimentation de matériaux fins devant les nombreux embâcles et qui uniformisent la couche sédimentaire;

— une gêne aux prélèvements opérables par l'activité halieutique.

Après la réalisation de l'opération de nettoyage, d'élagage, et de débroussaillage, peuvent être observés:

- une modification de la nature des fonds où deviennent plus nombreux les sédiments grossiers;

- une amélioration de la séquence d'écoulement radiersmouilles ;

- une réapparition ou un plus grand développement de la végétation aquatique ;

— un meilleur éclairement du milieu aquatique.

Selon l'état initial et selon l'importance des travaux ces transformations vont d'une perception visuelle évidente à une impression de changement plutôt faible.

Champigneulle (1976) a trouvé une augmentation de $2{ }^{\circ} \mathrm{C}$ du maxima de la température de l'eau du Scorff (56) après le nettoyage et l'élagage partiel de la rivière et de la végétation de la ripisylve. Il cite des études relatant le rôle de l'enlèvement de la végétation rivulaire sur les températures des petites rivières. Les peuplements d'invertébrés aquatiques sont 4 à 12 fois plus abondants dans les milieux courants à fond diversifiés que dans les zones sableuses engendrées par la présence d'embâcles.

Des suivis de populations de Truites dans 2 cours d'eau du Massif-Central (documents CSP, 1985-1988) ayant fait l'objet d'un nettoyage du lit et d'un entretien de la végétation des rives aboutissent à des constatations diverses. Sur le Vianon (19), un an après les travaux, le stock en place montre une densité de $87 \%$ supérieure à celle de l'année précédant la restauration et de $123 \%$ et $101 \%$ supérieure à celle observée en 2 stations amont à fort couvert végétal. Cette différence est due au seul recrutement de l'année. Par contre l'estimation des captures effectuées par les pêcheurs ne montre pas ni une meilleure réussite ni une meilleure fréquentation. Sur la Vienne (87) ont été aussi suivies les populations de truites (document CSP, 1988) avant le nettoyage du lit et des berges et 2 ans après. Alors qu'en 2 stations est observée une nette augmentation de la densité des juvéniles ( $65 \%$ et $114 \%$ ), par contre dans un autre secteur le phénomène inverse est constaté $(-41 \%)$. En plus la biomasse régresse partout de $11 \%, 28 \%$ et $49 \%$. Faute d'avoir investi suffisamment dans ces études celles-ci sont peu probantes et le jugement de l'effet positif des travaux repose davantage sur la perception du milieu : halieutisme facilité, réapparition de la végétation aquatique, que sur des données chiffrées sur les peuplements.

Il parait cependant utile de cerner davantage les conséquences de ces investissements financiers pour la faune ichtyaire dans divers types de cours d'eau afin de faire le 
bilan des effets positifs et négatifs. Parmi les paramètres à retenir, peuvent être cités :

- la restauration de la diversité du fond permettant à la fois l'existence de sites de reproduction que sont les plages de graviers placées dans les contrepentes, et la présence de zones d'abris fournies par les blocs (taille supérieure à $25 \mathrm{~cm}$ ) en remplacement des embâcles;

- le développement de l'éclairement et de la végétation aquatique et l'impact de celle-ci sur les poissons; impact qui peut s'exercer d'une manière directe (abris, support de nourriture ou de ponte) ou d'une manière indirecte (incidence sur la qualité de l'eau en milieu eutrophisé);

- l'évolution des peuplements ichtyaires : stocks, prélèvements halieutiques;

- les modifications de la qualité de l'eau : oxygénation et températures estivales qui quand elles sont trop élevées peuvent nuire à certaines espèces.

La diversité des rivières françaises liée à la variabilité des conditions naturelles et des activités humaines sur les bassins versants n'implique pas nécessairement que l'entretien de la ripisylve aboutisse partout à la même densité de végétation maintenue. Actuellement les travaux sont réalisés selon la perception qu'en a le maître d'œuvre ou le maître d'ouvrage.

\section{Conclusion}

En quelques décennies les aménagements du lit et des berges des cours d'eau ont globalement évolué. Très schématiquement on est passé de la chenalisation à un meilleur respect du tracé, des sections, et du profil naturel. Ceci a été obtenu par une limitation des coupures de méandres (coupures sèches éventuelles), par le maintien d'une berge intacte, par la création seulement là où c'est impératif de nouvelles sections à profils dissymétriques ou emboîtées par une protection des berges, par une conservation des séquences mouilles-radiers. La restauration des rivières qui consiste à enlever les embâcles du lit et à entretenir la ripisylve abandonnée constitue une solution plus récente mais qui a pu prendre, régionalement, beaucoup d'ampleur. Tous ces travaux avaient d'abord été motivés par des aspects purement hydrauliques locaux. Les conséquences désastreuses des chenalisations à la fois sur plan de l'hydraulicité, de la tenue des berges et des ouvrages, auxquelles s'est ajoutée petit à petit la prise en compte de la protection de l'environnement et de son utilisation ont engendré les réflexions ayant provoqué l'évolution des travaux. Cependant tout n'est pas parfait, loin s'en faut, pour ce qui est de la prise en compte de la protection de la faune ichtyaire. Les rivières recalibrées et reprofilées demeurent; leur restauration sous les seuls effets des éléments naturels peut être excessivement longue et le linéaire perdu risque de l'être pour très longtemps. En dépit des connaissances connues sur les conséquences néfastes pour le poisson, et malgré l'acquisition de certaines techniques de restauration (Mc CARTHY D.T., 1985), il ne semble pas que des investissements aient été faits pour améliorer les situations existantes. Parallèlement les travaux hydrauliques durs paraissent se développer sur les parties supérieures du réseau hydrographique, qui constituent très souvent d'excellentes zones de reproduction de la Truite ainsi que des réserves d'eau. Davantage de données méritent d'être récoltées sur les effets de l'entretien de la ripisylve afin d'éviter, le cas échéant, quelques erreurs eu égard au développement de la végétation aquatique ou à l'élévation de la température de l'eau dans les rivières à Truite. D'une manière plus générale les connaissances sur les populations de poissons doivent être améliorées et des études de suivi méritent d'être plus souvent engagées afin de mieux connaître l'incidence des divers travaux dans le milieu aquatique. Cela concerne aussi les aménagements affectant les débits.

Une gestion à l'échelle du bassin versant est nécessaire pour ne pas annuler là ce qui est sauvegardé ailleurs. Or l'examen au cas par cas des travaux en rivière quels qu'ils soient sans être replacé dans le cadre du bassin versant est de nature à aggraver les nuisances que subit la faune piscicole parfois en dehors de la zone où s'exercent les travaux.

Dans son rapport préparé dans le cadre des Assises Nationales de l'Eau, le groupe de travail sur la protection des milieux aquatiques a fort bien expliqué comment l'édifice vivant, lorsqu'il fonctionne correctement, assure une qualité convenable à l'eau permettant globalement de satisfaire l'ensemble des usages. La faune piscicole ne constitue que le maillon supérieur de toute l'échelle trophique qui, si elle n'est pas agressée, présente une diversité telle que les flux de matière et d'énergie aboutissent à une eau de bonne qualité. Les poissons participent aussi à la régulation des organismes producteurs qui lorsque le milieu est perturbé peuvent développer certaines proliférations. Conserver l'environnement aquatique en bonne santé constitue un moyen d'éviter de nombreux problèmes. Cela inclut aussi l'habitat du poisson, c'est-à-dire les compartiments physiques du cours d'eau et de son bassin versant pour lesquels une gestion isolée, ignorant les compartiments vivants, a conduit à des erreurs même sur le plan hydraulique. La diversité de l'habitat du poisson constitue pour les travaux en rivière un repère à prendre en compte et qui peut permettre de satisfaire à la fois les exigences des hydrauliciens et de l'environnement.

\section{Bibliographie}

ARNER D.H. (and al.) (1976). - Effects of Channelization of the Luxapalila River on Fish, Aquatic Invertebrates, Water Quality, and Furbearers. Office of Biological Services. Fish and Wildlife Service U.S. Department of the Interior. Washington D.C., 66 p.

Baudet J., Mouille J. (1981). - Aménagement de la Mortagne ; premier bilan écologique. Symposium, FAO-CECPI de Vichy. Colloque international sur la répartition des ressources ichtyologiques. 
Champigneulle A. (1976). - Le nettoyage du Scorff: son influence sur le peuplement végétal et le peuplement animal. D.A.A., spécialisation halieutique. E.N.S.A. Rennes, 22 p.

Clavel P. (1977). - Effets des extractions de matériaux alluvionnaires sur l'environnement aquatique dans les cours supérieurs de la Loire et de l'Allier. Thèse Université de Clermont $\mathrm{n}^{\circ} 510,141$ p., annexes.

CONSEIL Supérieur de LA PêCHE. - Documents internes DR Clermont 1985, Etude piscicole du Vianon, incidence du nettoyage du lit et des rives; premières observations par BRugel C., 1988, Compte-rendu des pêches électriques effectuées sur la Vienne en 1987, comparaison avec les résultats de 1983-1991, Compte-rendu des pêches électriques d'inventaire des populations piscicoles du Pontajou (43).

CRISP D.T. (1984). - Effects of impoundment and regulation of the river Tees at Cow Green upon fish populations in afferent tributaries and in the river immediately downstream of the deam. Proceedings of a Symposium of the European Inland Fisheries. Habitat Modification and Freshwater Fisheries, pp. 42-51.

CTGREF (1979), - Document remis à l'Administration départementale dans le cadre du suivi hydraulique des travaux d'aménagement du Litrou (63).

Cuinat R., Bomassi P., Carmie H. (1982), et collaborateurs. Conséquences hydrobiologiques et piscicoles de la chenalisation d'une petite rivière de Limagne. Association francaise de Limnologie. Congrès de Bordeaux, $6 \mathrm{p}$.

Demars J.J. (1985). - Repercussion of small hydro-electric power stations on population of Brown Trout (Salmo trutta) in rivers in the French Massif-Central. Proceedings of a Symposium of the European Inland Fisheries. Habitat Modification and Freshwater Fisheries, pp. 52-61.

Heland M. (1971). - Observations sur les premières phases du comportement agonistique et territorial de la Truite commune Salmo trutta L. en ruisseau artificiel. Ann. Hydrobiol., 2 (I), 33-46.

Hermansen H., Krog C. (1985). - A review of Brown Trout, Salmo trutta $\mathrm{L}$., spawning beds, indicating methods for their reestablishment in danish lowlands rivers. Proceedings of a Symposium of the European Inland Fisheries. Habitat Modification and Freshwater Fisheries, pp. 116123.

Huet M. (1949). - Aperçu des relations entre la pente et les populations piscicoles des eaux courantes. Revue Suisse d'Hydrologie, Vol. XI, Fasc. 3-4, 332-351.
Huet M., Timmermans J.A. (1976). - Influence sur les populations de poissons des aménagements hydrauliques de petits cours d'eau assez rapides. Station de Recherches des Eaux et Forêts de Groenendaal du Ministère de l'Agriculture. Travaux. Série D, $\mathrm{N}^{\circ} 46$.

Huet M., Timmermans J.A. (1976), - Fonctionnement et rôle d'un ruisseau frayères à truites. Station de Recherches des Eaux et Forêts de Groenendaal Ministère de l'Agriculture - Travaux - Série D, $\mathrm{n}^{\circ} 48$.

HUNT R.L. (1969). - Effect of Habitat Alteration on Production, Standing Crops and Yield of Brook Trout in Lawrence Creek, Wisconsin. Symposium on Salmon and Trout in Streams. Ed. Northcote, 281-312.

HUNT R.L. (1971), - Responses of a Brook Trout Population to Habitat Development in Lawrence Creek. Technical Bulletin, $\mathrm{N}^{\circ} 48$. Department of Natural Resources, Wisconsin.

LEwIS S.L. (1969). - Physical factors Influencing Fish Populations in Pools of a Trout Stream. Trans. Am. Fish. Soc.

Mc CARTHY D.T. (1985). - The adverse effects of channelization and their amelioration. Proceedings of a Symposium of the European Inland Fisheries: Habitat Modification and Freshwater Fisheries, pp. 83-97.

Milner N.J., Hemsworth R.J., Jones B.E. (1985). - Habitat Evaluation as a Fisheries Management Tool. J. Fish. Biol., $27,85-108$.

Ministère de l'Environnement et de la Protection des Risques Technologiques et Naturels Majeurs (1990). - Rapport du groupe de travail "Protection des milieux naturels aquatiques ». Document Assises Nationales de l'Eau.

Nunnaly N.R., Keller E. (1979). - Use of Fluvial Processes to minimize adverse Effects of Stream Channelization. Water Resources Research Institute of the University of North Carolina, $115 \mathrm{p}$.

TARPleE W.H., Darell E.L., Weber A.J. (1971). - Evaluation of the effects of channelization on Fish Populations in North Carolina's Coastal Plain Streams. North Carolina Wildlife Resources Commission, $23 \mathrm{p}$.

VerneauX J. (1981). - Les poissons et la qualité des cours d'eau. Annales scientifiques de l'Université de Franche-Comté. Biologie Animale, $4^{\mathrm{e}}$ série, fasc. 2, 33-41.

WhITE R.J., BRYNILDSON O.M. (1967). - Guidelines for Management of Trout Stream Habitat in Wisconsin. Technical Bulletin $N^{\circ} 39$. Department of Natural Resources, $64 \mathrm{p}$. 